\title{
Foot and Ankle
}

REVIEW ARTICLE

\section{Chosen Conservative Treatments on the Symptoms of Calcaneal Spur: A Short Review}

\section{Pawel Lizis*}

\section{Department of Education and Health Protection, Holy Cross College, Kielce, Poland}

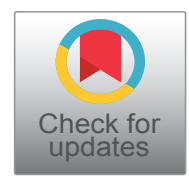

*Corresponding author: Pawel Lizis, Department of Education and Health Protection, Holy Cross College, Mielczarskiego 51,25-709 Kielce, Poland, E-mail: pawel_lizis@poczta.onet.pl

\begin{abstract}
Background: Calcaneal spur (CS) occurs when calcium deposits build up on the underside of the heel bone, a process that usually lasts for many months. CS often causes foot muscle strain, inflammation of the plantar fasciitis, repeated tearing of the membrane that covers the heel bone and pain. CS decreases physical activity, social capacity, reducing the quality of life, and becomes the cause of frequent absence from work due to sickness. It is a disease commonly appearing in adults, and it is also a serious social problem. Physiotherapy is an alternative method for pharmacology and surgery. It includes kinesiotherapy, orthoses, corticosteroid therapy, and electrotherapy, but their efficacy remains controversial. The aim of this manuscript was a short review of the effectiveness of therapeutic ultrasound (US) and extracorporeal shock wave therapy (ESWT) on the symptoms of CS.
\end{abstract}

Methods: The study was designed as a short review identified from the Cochrane Controlled trials register, MEDLINE, EMBASE, CINAHL, Pubmed, Science Direct, Web of Science and Polish National Library from January 2000 till December 2016. The specific and unified inclusion criteria were: People diagnosed with unilateral X-ray on the CS at the minimum age of 40 , and those patients who felt pain under the calcaneal tuber persisting longer than 8 months, the pain measured after daily activity by visual analog scale (VAS), numeric pain rating scale (NPRS) the term of research which was executed at the end of the treatment (from week 3 to months 72). In this short review the following data was extracted: Pain, Ankle-Hindfoot Scale, Roles and Maudsley Score, SF-12 health status questionnaire.

Results: Systematic short reviews showed that especially therapeutic ultrasound (US) as well as extracorporeal shock wave therapy (ESWT) would be appropriate for the treatment of the patients suffering from CS. In this short review only two studies compared the effectiveness of ESWT versus US on the symptoms of the CS. The findings showed that ESWT was clinically more efficient for reducing plantar pain and for functional improvement of foot health status in people suffering from chronic CS.
Conclusion: US and especially ESWT must be considered as the first step of treatment for CS associated with inflammatory of plantar fasciitis. In summary, the results of the short review provide the evidence that patients with CS can obtain significant health benefits to foot care with ESWT.

\section{Keywords}

Calcaneal spur, Extracorporeal shock wave therapy, Therapeutic ultrasound, Foot

\section{Introduction}

Patients with calcaneal spur (CS) associated with inflammatory of plantar fasciitis, in whom symptoms cannot be controlled after 9-12 months of conservative management, may become candidates for surgery [1]. Plantar fascial release, including the first layer of intrinsic muscles, has been shown to be effective in recalcitrant cases. Endoscopic plantar fasciotomy is also a reasonable option where conservative therapy has failed. In nerve entrapment, if conservative measures are ineffective after six to 12 months, surgical decompression should be considered [2-4]. And in recalcitrant cases, surgery to remove the Haglund deformity may be necessary [5].

Physiotherapy is an alternative method for pharmacology and surgery in people suffering from the calcaneal spur (CS). Conservative treatment of the CS consists in various physiotherapy treatments, e.g., kinesiotherapy, orthoses, corticosteroid therapy, iontophoresis, laser, ultrasound (US), phonophoresis, and lately increasingly used extracorporeal shock wave therapy (ESWT). However, their but their efficacy remains controversial [6-8]. The electrotherapeutic method is primarily based on the use of thermal, physical-chemical

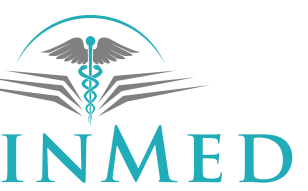

INTERNATIONAL LIBRARY

Citation: Lizis P (2018) Chosen Conservative Treatments on the Symptoms of Calcaneal Spur: A Short Review. Int J Foot Ankle 2:006. 
and mechanical properties of ultrasound waves. The heat produced in the tissues by electrotherapy leads to various changes typical of this energy: Congestion, increased metabolism, increased the extensibility of collagen fibers, increased enzyme activity, decreased muscle tone, changes in nerve conduction, pain relief, decreased joint stiffness [9]. The aim of this manuscript was a short review of the effectiveness of US and ESWT on the symptoms of CS.

\section{Material and Methods}

The study was designed as a short review identified from the Cochrane Controlled trials register, MEDLINE, EMBASE, CINAHL, Pubmed, Science Direct, Web of Science and Polish National Library from January 2000 till December 2016. The specific and unified inclusion criteria were: People diagnosed with unilateral $\mathrm{X}$-ray on the $\mathrm{CS}$ at the minimum age of 40 , and those patients who felt pain under the calcaneal tuber persisting longer than 8 months, the pain measured after daily activity by visual analog scale (VAS), numeric pain rating scale (NPRS) the term of research which was executed at the end of the treatment (from week 3 to months 72). In this short review the following data was extracted: Pain, Ankle-Hindfoot Scale, Roles and Maudsley Score, SF-12 health status questionnaire.

\section{Literature Review}

Łukowicz, et al. [8] compared analgesic effects of low level laser therapy and phonophoresis on pain in fifty-six patients suffering from the CS. Subjects were divided into two groups. The first group of 23 patients was subjected to laser therapy $\left(830 \mathrm{~nm}, 300 \mathrm{~mW}, 4-6 \mathrm{~J} / \mathrm{cm}^{2}\right)$ and the second (33 subjects) to phonophoresis procedures $\left(0.8 \mathrm{MHz}, 0.8-1 \mathrm{~W} / \mathrm{cm}^{2}\right)$. Both groups received 10 treatments, once a day, 5 per week. Each treatment didn't exceed 5 minutes. Pain was measured by Visual Analog Scale (VAS) and on Laitinen scale twice-at baseline and after the last treatment. A significant decrease in pain intensity was observed in both groups compared to baseline values, $p<0.05$, however no significant difference between groups fund after the end of treatment.

Straburzyńska-Lupa and Kornacka [9] assessed the analgesic effect of different doses of US in 34 patients suffering from CS. For this purpose, group I was applied a higher dose $\left(1.0-1.6 \mathrm{~W} / \mathrm{cm}^{2}\right.$ - during the first week; $1.6-1.8 \mathrm{~W} / \mathrm{cm}^{2}$ - during the second week, and during the last session the maximum dose of $\left.2.0 \mathrm{~W} / \mathrm{cm}^{2}\right)$. Group II received a lower dose $\left(0.6-0.8 \mathrm{~W} / \mathrm{cm}^{2}\right.$ - during the first week, 0.8-1.0 W/ $\mathrm{cm}^{2}$-during the second week). The pain was assessed on VAS at baseline and after 10 sessions. It turned out that in group I the pain levels decreased after 5 sessions, while in group II after 10 sessions. These research results were the basis for the authors' thesis that the optimal analgesic dose of ultrasound for patients with heel spurs is $1.0-1.6 \mathrm{~W} / \mathrm{cm}^{2}$. The authors noted that higher doses resulted in increased pain in the area around calcaneus bone which probably was caused by the lack of tolerance for the maximum dose- $2.0 \mathrm{~W} /$ $\mathrm{cm}^{2}$; that is why the maximum dose should not be used in the treatment of pain caused by the CS.

Zanon, et al. [10] evaluated a high power, continuous-mode US efficacy ion chronic plantar fasciitis. Twenty-two participants were randomized into Group 1 (kinesiotherapy + US off) or Group 2 (kinesiotherapy + US effective). Kinesiotherapy involved five stretching exercises, each one lasting three minutes, for the leg posterior musculature and plantar fascia. US was applied with the following parameters: Continuous mode, basic frequency of $1 \mathrm{MHz}$, power $2.0 \mathrm{~W} / \mathrm{cm}^{2}$, applied during three minutes on each region (calcaneus medial tuberosity and on the $2 \mathrm{~cm}$ distal to tuberosity). All the process, intervention and evaluations demanded 15 sessions, constituting a total of five weeks. Functional evaluation performed on the first and the last sessions used a questionnaire employed by American Orthopedic Foot and Ankle Society (AOFAS). The pain on VAS was also assessed at the first and at the last session. The scores obtained from AOFAS questionnaire showed a post-treatment improvement, that is, an increase of scores for both groups, with no significant difference to each other. The pain levels at the three-evaluation point showed that both groups presented a significant improvement during the 15 procedure sessions. The pain level average at the end of treatment was statistically equivalent for groups 1 and 2 .

Boerner, et al. [11] compared the analgesic efficacy of US in a dose-dependent treatment $\left(0.8 \mathrm{~W} / \mathrm{cm}^{2}\right.$ and $1.2 \mathrm{~W} / \mathrm{cm}^{2}$ ) in 40 patients with CS. The pain was measured on VAS. The study found that both doses reduce the level of pain, although the dose of $0.8 \mathrm{~W} / \mathrm{cm}^{2}$ was more effective. In addition, it was found that at a dose of $0.8 \mathrm{~W} / \mathrm{cm}^{2}$ the level of pain decreased by $34 \%$ after 5 sessions, by $50 \%$ after 10 sessions, and by $71 \% 4$ weeks after the completion of therapy. While at a dose of 1.2 $\mathrm{W} / \mathrm{cm}^{2}$ the level of pain decreased by $24 \%$ after 5 sessions, by $24 \%$ after 10 sessions, and by $66 \% 4$ weeks after the completion of therapy.

Jasiak-Tyrkalska, et al. [12] compared the efficiency a single session of US and phonophoresis on CS. Forty patients were randomized into two equal groups. Group A was treated using phonophoresis with ketoprofen gel. Group B was treated using US. Both groups received a dose $0.5 \mathrm{~W} / \mathrm{cm}^{2}$ during the first session and progress to $1 \mathrm{~W} / \mathrm{cm}^{2}$, at a $20 \%$ duty cycle and the frequency of 1 $\mathrm{MHz}, 8$ minutes per session. Pain on VAS was assessed. The study showed that phonophoresis is more effective in reducing pain than US in patients with CS. The study showed the reduction of the pain level at $p<0.001$ in the phonophoresis treatment group, and the level of $p$ $<0.01$ in the US treatment group.

Twarowska, Niemrzycka [13] compared the effects of 10 sessions of US and selected techniques of manu- 
al therapy on pain level and functional state in patients with the CS. Twenty-two patients (14 females and 8 males) with CS were included in the study. They were randomly assigned to two groups ( $A$ and $B$ ) with different therapies administered. Group A was treated with US combined with soft tissue therapy, while group B was subjected to manual therapy only. US was administered using $0.8 \mathrm{~W} / \mathrm{cm}^{2}$ wave intensity, at a frequency of 1 $\mathrm{MHz}$, ERA (Effective Radiating Area) of $5 \mathrm{~cm}^{2}$, BNR (Beam Non-Uniformity Ratio) below 5 -minute ultrasound exposure and a continuous output ( $100 \%$ duty cycle). UItrasound gel was applied in the therapy. The dynamic method was used, and circular moves were performed to transmit ultrasonic waves to the calcaneal tuberosity on the plantar side.

Soft tissue therapy consisted of a dynamic massage of deep tissues and a transverse massage. Massages were given to the patients lying in a supine position according to the following procedure: 1 . Three-minute massage of the plantar fascia (neutral position of the foot) where sliding moves along the plantar fascia (from toes to the heel) were performer, 2. Two-minute massage of the stretched plantar fascia. The stretch was achieved through a passive extension of the foot phalanges, 3 . Five-minute transverse massage of the plantar fascia (neutral position of the foot) in regions of the greatest tension and pain.

The following research tools were employed in the study: 1. Numeric Pain Rating Scale (NPRS) used for assessing pain intensity before each therapy session, upon arising in the morning (first step), at the end of the day and 3 weeks post the therapy; 2 . Ankle-Hindfoot Scale (AHFS); 3. Functional tests (standing and walking on tiptoe and heels) assessed with NPRS; 4. Data gathered from the radiography image interpretation and an interview carried out in compliance with the patient examination form. The research tools were used on an admission day and after a series of therapy sessions. Furthermore, pain intensity was rated prior to each session and 3 weeks post the therapy. A decrease in pain intensity was observed in both groups. After 10 therapy sessions, it decreased by an average of 3.72 points $(59 \%)$ in group A and by 3.55 points (75\%) in group B on an NPRS. Three weeks post therapy, group $A$ exhibited a decrease by an average of 3.91 points $(62 \%)$, while group B reported a decrease by 3.82 points ( $83 \%$ ) compared to baseline values. Pain intensity measured during the first step in the morning and at the end of the day decreased in both groups. The difference between the groups was not significant. The values of particular components of Ankle-Hindfoot Scale (AHFS) which indicated the greatest difference was noted with regard to hindfoot pain. On average, group A and group B scored 16.36 points and 15.46 points, respectively. No differences were found in the sagittal motion, ankle-hindfoot stability and foot alignment. The differences in both groups were significant at $p<0.001$. The findings showed, that a series of
10 therapy sessions is enough to improve pain and functional capacity outcomes; however, it will never result in complete pain relief.

Another an alternative to the surgical treatment of the CS is extracorporeal shock wave therapy (ESWT) that is a non-invasive method, it causes micro breaks in avascular or poorly-vascularized tissue thus stimulating appropriate revascularization and stem cell growth [14].

The review of the subject literature shows that the analgesic efficacy of ESWT with different physical characteristics and over a different period of time concerning was investigated.

Metzner, et al. [15] used ESWT in 63 patients with plantar aponeurosis inflammation. Each patient got 1000 impulses of ESWT, the stream density of the emitted energy was $0.35 \mathrm{~mJ} / \mathrm{mm}^{2}$. The pain on VAS was examined 6 weeks, 18 months and 72 months, after the end of ESWT. It turned out that the pain decreased of $30 \%$ at $81 \%$ of the patients after 6 weeks, at $88 \%$ of the patients after 18 months, and at $96 \%$ of the patients in the last examination 72 months after the end of ESWT. On the basis of the results the authors concluded that the used ESWT doses successfully decreased the pain, and the treatment effects gave satisfying long-term results.

Yalcin, et al. [16] examined the effects of ESWT on calcaneus bone spurs and the correlation between clinical outcomes and radiologic changes. The study involved 108 patients with heel pain and radiologically diagnosed heel spurs. All patients underwent ESWT once a week for 5 weeks at the clinic. Each patient received 2,000 impulses of shock waves, starting with $0.05 \mathrm{~mJ} /$ $\mathrm{mm}^{2}$ (1.8 bar) and increasing to $0.4 \mathrm{~mJ} / \mathrm{mm}^{2}$ (4.0 bar). The standard radiographies of the affected heels were obtained before and after the therapy. Clinical results demonstrated excellent (no pain) in $66.7 \%$ of the cases, good $(50 \%$ of pain reduced) in $15.7 \%$ of the cases, and unsatisfactory (no reduction in pain) in $17.6 \%$. After five ESWT treatments, no patients who received shock wave applications had significant spur reductions, but 19 patients $(17.6 \%)$ had a decrease in the angle of the spur, 23 patients $(21.3 \%)$ had a decrease in the dimensions of the spur, and one patient had a broken spur. Therefore, results showed no correlation between clinical outcome and radiologic changes. The present study supports the finding that even with no radiologic change after ESWT therapy, the therapy produces significant effects in reducing patients' complaints about CS.

Moretti, et al. [17] evaluated the analgesic efficacy of low doses of ESWT for foot plantar fascia inflammation accompanying CS in 54 runners-athletes. The subjects received a weekly shockwave of 1000 impulses, $0.06 \mathrm{~mJ} / \mathrm{mm}^{2}$ energy density. The pain was assessed on VAS. ESWT treatment continued for four weeks, then the patients were examined after 45 days, and 6 and 24 months after the last session. The clinical results were 
excellent in $59 \%$ of cases, good in $12 \%$ of cases, satisfactory in $21 \%$ and clearly unsatisfactory in $8 \%$. The low-energy ESWT seems to be a good means to treat inflammation of foot plantar fascia in runners, because the resulting improvement persisted for 24 months from the end of ESWT.

Hammer, et al. [7] assessed the analgesic efficacy of ESWT in 57 patients with painful chronic inflammation of the plantar fascia. Patients treated with ESWT were given 3000 impulses of shocks with energy density of 0.2 $\mathrm{mJ} / \mathrm{mm}^{2}$ at weekly intervals. Two years after the end of treatment the level of pain on a VAS scale in patients treated with ESWT decreased 94\%.

Cosentino, et al. [18] evaluated the analgesic efficacy ESWT at 60 patients with calcaneal enthesophytosis. Patients were randomly assigned to two equal groups. The ESWT (group 1) received six treatments (one every 7-10 days), each treatment consisting of 1200 shocks with a frequency of 120 shocks $/ \mathrm{min}$; the energy density used varied from 0.03 to $0.4 \mathrm{~mJ} / \mathrm{mm}^{2}$ and the control (group 2 ) went through the identical process but energy density was simulated $\left(0 \mathrm{~mJ} / \mathrm{mm}^{2}\right)$. The results revealed the significant reduction of pain in the ESWT (group 1). In the control (group 2) no significant decrease of VAS was seen.

Wang, et al. [19] evaluated the result of shockwave treatment for plantar fasciitis. 149 participants were randomly allocated to ESWT or control group. In the ESWT group, patients received 1500 impulses of shockwaves at $16 \mathrm{kV}$ (energy flux density, $0.32 \mathrm{~mJ} / \mathrm{mm}^{2}$ ) to the affected heel as a 1-time treatment. Treatments were performed on an outpatient basis using local anesthesia with $2 \%$ xylocaine. The area of treatment was focused with a control guide on the machine, and surgical lubricant was placed on the skin in contact with the shockwave tube. The patient's vital signs and local discomfort were monitored throughout the course of treatment. The treated area was inspected for local swelling, ecchymosis, or hematoma immediately after the treatment. Patients were sent home with a non-narcotic analgesic such as acetaminophen; NSAIDs were not prescribed. Patients in the control group were treated with NSAIDs, orthotics, physical therapy, an exercise program, or a local cortisone injection. Patients were initially treated with a single modality (NSAIDs). Additional modalities such as physical therapy, orthotics, and an exercise program were subsequently prescribed, either singularly or in combination, if the initial modality failed to provide satisfactory results or if patients developed recurrence of symptoms. A local cortisone injection with $0.5 \mathrm{~mL}$ of betamethasone $(7 \mathrm{mg} / \mathrm{mL})$ and $1.0 \mathrm{~mL}$ of $2 \%$ xylocaine was given only to patients with severe heel pain. Follow-up examinations were performed independently by one of the coauthors, who was blinded to patient treatment status. Pain intensity was recorded on a 10-point Visual analog scale, with 0 for no pain and 10 for severe pain. Radiographs of the heel were obtained before treatment and at the most recent follow-up examination. The clinical outcomes were rated as excellent, good, fair, or poor. An excellent result was defined as having no heel pain on all activities of daily living, including sports; a good result as having less than $50 \%$ of the original heel pain on certain activities, including sports; a fair result as having $50 \%$ to $75 \%$ of the original heel pain on certain activities; and a poor result as having $75 \%$ or more of the original heel pain. Patients were evaluated at 60 to 72 months (shockwave group) or 34 to 64 months (control group) with a 100-point scoring system including 70 points for pain and 30 points for function. Before treatment, the groups showed no significant differences in the scores for pain and function. After treatment, the shockwave group showed significantly better pain and function scores as compared with the control group. The overall results were $69.1 \%$ excellent, $13.6 \%$ good, $6.2 \%$ fair, and $11.1 \%$ poor for the shockwave group; and $0 \%$ excellent, $55 \%$ good, $36 \%$ fair, and $9 \%$ poor for the control group $(p<0.001)$. The recurrence rate was $11 \%(9 / 81$ heels) for the shockwave group versus $55 \%$ ( $43 / 78$ heels) for the control group ( $p$ $<0.001$ ). There were no systemic or local complications or device-related problems.

Shaheen [20] evaluated 46 patients with unilateral plantar fasciitis. The patients were randomly divided into two equal groups to receive either active treatment (group) or placebo regimens (group) according to the computer generated random numbers list. Low-energy radial extracorporeal shock wave therapy (rESWT) was provided with energy flux density $0.16 \mathrm{~mJ} / \mathrm{mm}^{2} ; 2000$ impulses; 2.5 bars and frequency of $8 \mathrm{~Hz}$ without local anesthesia. Group I received a total 3 treatments $(3 \times$ 2000 impulses) given at weekly interval. While group II received the identical treatment protocol; however; shockwaves were prevented from entering the patient's foot by thin foam cushion placed on the therapy head. The cushion was put in place prior to the patient's arrival in the treatment room to maintain blinding. A new cushion was used with each treatment session. Pain and limitation of foot function were measured by VAS and Ankle-Hind Foot Scale (AHFS) respectively. The measurements were performed at a base line, after 3 weeks and after 6 weeks of follow up (after the completion of treatment). The results revealed the significant reduction of pain in both groups after 3 weeks of treatment ( $p<0.000$ and 0.005 respectively). However, there was a significant improvement in foot function in group both after 3 weeks of treatment and 6 weeks of follow up $(p=$ 0.000 ). Despite the small number of patients in this trial, low-energy rESWT was an effective non-invasive treatment method for chronic plantar fasciitis and may help the patients to avoid surgery.

Theodore, et al. [21] evaluate further the clinical effectiveness of high-energy ESWT for the treatment of plantar fasciitis during a single therapeutic session. The 
participants were randomized in 1:1 ratio to the Active Group ( $n=76$ ), which received ESWT or to the Control Group ( $n=76$ ), which received a sham treatment. All study patients, including the Control Group, were given a medial calcaneal nerve block using $5 \mathrm{~mL}$ of $1 \%$ xylocaine 15-20 minutes prior to the procedure. All patients were placed in the prone position and ultrasound visualization of the proximal plantar fascia origin was performed. The Active Group received 3800 shocks (3500 at $0.36 \mathrm{~mJ} / \mathrm{mm}^{2}$ ) for a total of $1300 \mathrm{~mJ} / \mathrm{mm}^{2}$. The Control Group went through the identical process but had a thin air cushion placed on the therapy head to prevent shock wave penetration into the foot. The air cushion was placed prior to the patient entering the treatment room to further ensure blinding. All patients were evaluated at pretreatment and at 3-5 days, 6 weeks, 3 months, 6 months, and 12 months post-treatment. Patients were assessed by means of the visual analog scale (VAS) for pain Turing the first few minutes of walking in the morning, pain with normal activity during the day, pain with leisure time/sport-related physical activity, and pain prior to going to bed for the evening. A Roles and Maudsley Score, SF-12 health status questionnaire, Ankle-Hindfoot Scale (AHFS), and physical examination, including pressure threshold measurement were also used. Evaluations were performed at each center by an independent physician who was blinded to the treatment status of the patients. In the Active Group, pain decreased at 3 months post-treatment $(p=0.0001)$, resulting in a mean percent improvement of $57 \%$. Also, in the Control Group, pain decreased at 3 months post-treatment ( $p$ $=0.0001$ ), resulting in a mean percent improvement of $47 \%$. The proportion of patients achieving at least a $60 \%$ improvement (clinical success) in pain during the first few minutes of walking in morning was compared between the two groups at 3 months. In the Active Group, $56 \%(41 / 73)$ of the patients achieved a $60 \%$ reduction in their VAS pain score compared to $45 \%(33 / 73)$ in the Control Group. The difference between the groups, with the numbers available, did not reach statistical significance ( $p=0.1885)$. The secondary efficacy points included the Roles and Maudsley Score, which is a fourpoint patient self-assessment of pain and limitations of activity. At 3 months post-treatment, the Active Group had $62 \%$ (45/73) of the patients change from a fair/poor response at baseline to an excellent/good assessment, compared to $40 \%$ (29/73) for the Control Group. This comparison was statistically significant ( $p=0.0327$ ). Other secondary points, including Ankle-Hindfoot Scale (AHFS) and SF-12 health status questionnaire, did not show statistically significant differences between the two groups. Numerical trends in favor of the Active Group, though not statistically significant, were observed in the AHFS pain score and the SF-12 physical component score.

Krukowska, et al. [22] compared the analgesic efficacy of US and ESWT in patients with CS. Forty-seven pa- tients were randomly assigned into two groups using a simple randomization: Group 1-US (a series of ten treatments) and Group 2-radial shock wave (rESWT; series of four treatments). US was performed using a labile technique, targeting the calcaneal tumor and attachment of the plantar aponeurosis (pain points); the power setting was $1.5 \mathrm{~W} / \mathrm{cm}^{2}, 80 \%$ fill factor, at a frequency of $1 \mathrm{MHz}$ (head $4 \mathrm{~cm}^{2}$ ). The duration of a single treatment was $4 \mathrm{~min}$, and the coupling substance used was paraffin oil. Treatment sessions were performer daily Monday through Friday for a period of 2 weeks in a series of ten treatments. The rESWT was performed on the affected area using 2000 strokes with a frequency of $10 \mathrm{~Hz}$ and a capacity of 2.5 bar. Therapy took place for 2 weeks in a series of four treatments with a 3-day interval between treatments. In all patients, pain intensity on Laitinen and VAS was assessed three times: Before therapy, after the first and second weeks of treatment. However, a decrease in pain sensation was reported in all test intervals, and its largest decrease occurred in both groups within 1 week of beginning treatment. More dynamic change in this period was recorded in Group 1 . The conclusion is that while US and rESWT showed significant analgesic efficacy in patients with calcaneal spur, fewer ESWT sessions are needed than US sessions for effective relief. Finally, the results suggest that the rESWT has greater analgesic efficacy. Lizis, Hudakova [23] compared the ESWT and the US influences on the improvement of the feet health status of the patients with CS whose heel pain lasted longer than 6 months. The patients treated with ESWT got the altogether dose of 7000 impulses of shock waves, energy flux density $0.4 \mathrm{ml} / \mathrm{mm}^{2}$ during 5 treatments performed once a week for 5 weeks. The patients treated with US got the dose of $0.8 \mathrm{~W} / \mathrm{cm}^{2}$ during 10 treatments performed three times a week. In both groups the authors noted the significant improvement in the feet health status. However, the patients treated with ESWT had significantly greater pain decrease and life quality improvement, and those benefits were still present 3 months after the treatment.

\section{Conclusion}

Systematic reviews and own experiences showed that the therapeutic US, as well as ESWT would be appropriate for the treatment of the patients suffering from CS. The collected data show a significant percentage of success in a short-term period as well as in a longterm period. From literature analysis, it arises that the variability in results regarding US and ESWT depends on treatment modalities (waves intensity, number of impulses, low or high energy, use or less of anaesthesia) and on the duration of pathology. US and ESWT are used for pain relief and for improving the quality of life and physical function in patients suffering from CS associated with inflammatory of plantar fasciitis.

In this short review only two studies compared the effectiveness of ESWT versus US on the symptoms of 
the CS. The findings showed that ESWT was clinically more efficient for reducing the plantar pain and for the functional improvement of foot health status in people suffering from chronic CS. Furthermore, the therapeutic effect persists for long-term period post the treatment, which indicates the high effectiveness of the ESWT treatment. Therefore, ESWT has several advantages and should be considered as an effective and safe tool in the treatment of chronic CS. As an alternative to a surgery it is a noninvasive technology, which has considerably less complications. Finally, ESWT can help in reducing pain in patients suffering from CS, loss of time at work and health care costs associated with prolonged treatments and surgery.

US and especially ESWT must be considered as the first step of treatment for heel spur associated with inflammatory of plantar fasciitis, due to the effectiveness, the reduced costs and the safety of the procedure. Systematic reviews may be valuable for physicians, physiotherapists and patients with CS in terms of the selection of the most appropriate treatment on the basis of patients' preferences and convenience.

\section{Conflict of Interest}

The authors declare that they have no conflict of interest.

\section{Acknowledgement}

None.

\section{References}

1. Skinner HB (2000) Current Diagnosis \& Treatment in Orthopedics. ( $2^{\text {nd }}$ edn), McGraw-Hill Companies, Inc 2000: 444-446.

2. Urovitz EP, Birk-Urovitz A, Birk-Urovitz E (2008) Endoscopic plantar fasciotomy in the treatment of chronic heel pain. Can J Surg 51: 281-283.

3. Tu P, Bytomski JR (2011) Diagnosis of heel pain. Am Fam Physician 84: 909-916.

4. Sadek AF, Fouly EH, Elian MM (2015) Lateral plantar nerve release with or without calcaneal drilling for resistant plantar fasciitis. J Orthop Surg (Hong Kong) 23: 237-240.

5. Thomas JL, Christensen JC, Kravitz SR, Mendicino RW, Schuberth JM, et al. (2010) The diagnosis and treatment of heel pain: A clinical practice guideline-revision. J Foot Ankle Surg 49: S1-S19.

6. Robertson V, Baker K (2001) A review of therapeutic ultrasound: Effectiveness studies. Phys Ther 81: 1339-1350.

7. Hammer DS, Adam F, Kreutz A, Kohn D, Seil R (2003) Extracorporeal shock wave therapy (ESWT) in patients with chronic proximal plantar fasciitis: A 2-year follow-up. Foot Ankle Int 24: 823-828.

8. Łukowicz M, Weber-Rajek M, Ciechanowska K, Włodarkiewicz A (2009) Evaluate the effectiveness of low-energy laser therapy and iontophoresis in the treatment of heel spur symptoms. Acta Bio-Optima et Informatica Medica 15: 340-343.

9. Straburzyńska-Lupa A, Kornacka A (2005) Ultrasound therapy in the treatment of calcar pedis-Own experiences. Ortophedic Traumatology Rehabilitation 7: 79-86.
10. Zanon RG, Brasil AK, Imamura M (2006) Continuous ultrasound for chronic plantar fasciitis treatment. Acta Ortop Bras 14: 137-140.

11. Boerner E, Toruń-Kotarska E, Kuciel-Lewandowska J (2009) Comparision of the performance of ultrasound in a dose- dependent in the treatment of calcaneal spurs. Acta Bio-Optica et Informatica Medicina 15: 230-233.

12. Jasiak-Tyrkalska B, Jaworek B, Frańczuk B (2007) To evaluated the efficacy of two physical treatments in comprehensive physiotherapy plantar calcaneal spurs. Polish Journal of Physiotherapy 7: 145-154.

13. Twarowska N, Niemierzycka A (2016) Effectiveness of using ultrasound therapy and manual therapy in the conservative treatment of calcaneal spur-pilot study. Progress of Rehabilitation 2: 63-74.

14. Wang CJ, Wang FS, Huang CC, Yang KD, Weng LH, et al. (2005) Treatment for osteonecrosis of the femoral head: Comparison of extracorporeal shock waves with core decompression and bone-grafting. J Bone Joint Surg Am 87: 2380-2387.

15. Metzner G, Dohnalek C, Aigner E (2010) High-energy Extracorporeal Shock-Wave Therapy (ESWT) for the treatment of chronic plantar fasciitis. Foot Ankle Int 31: 790-796.

16. Yalcin E, Keskin Akca A, Selcuk B, Kurtaran A, Akyuz M (2012) Effects of extracorporal shock wave therapy on symptomatic heel spurs: A correlation between clinical outcome and radiologic changes. Rheumatol Int 32: 343-347.

17. Moretti B, Garofalo R, Patella V, Sisti GL, Corrado M, et al. (2006) Extracorporeal shock wave therapy in runners with a symptomatic heel spur. Knee Surg Sports Traumatol Arthrosc 14: 1029-1032.

18. Cosentino R, Falsetti P, Manca S, De Stefano R, Frati E, et al. (2001) Efficacy of extracorporeal shock wave treatment in calcaneal enthesophytosis. Ann Rheum Dis 60: 10641067.

19. Wang CJ, Wang FS, Yang KD, Weng LH, Ko JY (2006) Long-term Results of extracorporeal Shockwave Treatment for Plantar Fasciitis. Am J Sports Med 34: 592-596.

20. Shaheen Afaf AM (2011) Low-Energy Radial Extracorporeal Shock Wave Therapy for Chronic Plantar Fasciitis: A Randomized Control Trial. World Applied Sciences Journal 12: $10-15$.

21. Theodore $\mathrm{GH}$, Buch $\mathrm{M}$, Amendola A, Bachmann C, Fleming LL, et al. (2004) Extracorporeal shock wave therapy for the treatment of plantar fasciitis. Foot Ankle Int 25: 290-297.

22. Krukowska J, Wrona J, Sienkiewicz M, Czernicki J (2016) A comparative analysis of analgesic efficacy of ultrasound and shock wave therapy in the treatment of patients with inflammation of the attachment of the plantar fascia in the course of calcaneal spurt. Arch Orthop Trauma Surg 136: 1289-1296.

23. Lizs P, Hudáková Z (2016) Influence of two conservative treatment methods on foot health status in men with chronic calcaneal spur: A randomized controlled study. Elsevier Kontakt 18: e36-e41. 